\title{
Low Contrast Enhancement Technique for Color Images using Interval-Valued Intuitionistic Fuzzy Sets with Contrast Limited Adaptive Histogram Equalization
}

\author{
Reegan Jebadass J • Balasubramaniam $\mathbf{P}^{\dagger}$
}

Received: date / Accepted: date

\begin{abstract}
This work introduces a program to enhance images taken in low light. Fuzzy set theory is creating a significant shift in image processing. Interval-valued intuitionistic fuzzy sets (IVIFS) based on intuitionistic fuzzy sets constructed from fuzzy sets are used to enhance images taken in low light. In the proposed method, first the given low light image is fuzzified by normal fuzzification. Then the fuzzified image is converted to an interval-valued intuitionistic fuzzy image. This image will be proposed enhanced image after applying the contrast limited adaptive histogram equalization (CLAHE). The experimental results reveal that the proposed method gives better results when compared with other existing methods like histogram equalization (HE), CLAHE, brightness preserving dynamic fuzzy histogram equalization (BPDFHE), histogram specification approach (HSA). Based on the performance analysis like entropy and correlation coefficient (CC), the proposed method gives better results.
\end{abstract}

Keywords Histogram Equalization · Image Enhancement · Interval-Valued Intuitionistic Fuzzy Sets.

Mathematics Subject Classification (2010) 68U10 - 94D05

\section{Introduction}

Image processing is the field of study in which algorithms operate on input images to produce output images. Image enhancement involves transforming an input image into another image so as to improve its visual appearance. An example of enhancement is to brighten an originally dark image or to increase the contrast of an image to make the details more visible. Another example is to detect the intensity edges of an image in order to highlight the boundaries of objects or to

Department of Mathematics,

The Gandhigram Rural Institute (Deemed to be University)

Gandhigram-624 302, Tamil Nadu, India

E-mail: reeganmail@gmail.com,

E-mail: balugru@gmail.com ${ }^{\dagger}$

$\dagger$ corresponding author. 
colorize a gray scale image to make the different data values more distinguishable to a human observer.

Low illumination/low contrast images are enhanced by computer vision and pattern recognition. Over the past few decades, histogram equalization (HE), fuzzy logic-based methods, etc have played a major role in improving images taken in low light. To enhance the quality of an image for better visual perception, many HE based methods [1-8] have been proposed. Puer et al [7] introduced adaptive histogram equalization (AHE) which is an excellent contrast enhancement method for both natural images and medical and other initially non visual images. Jianga et al [8] introduced an approach to specifying the correct histogram profile, which means that the intensity values of the image are adjusted accordingly and the output brightness is maintained close to the input image. In particular, the equilibrium profile is designed by finding the equilibrium control threshold by performing the integration of the rectangular and triangular sections. Patia and Ogate [9] proposed the technique of image enhancement based on a statistical analysis of DCT coefficients. This method would arrive at the DC coefficient and AC coefficients of a DCT module separately for two different measurement factors. Pisano et al [10] introduced a method to improve the detection of simulated speculations on dense mammograms. The purpose of this project is to determine whether CLAHE improves the detection of simulated speculations on dense mammograms. In 2003, Reza [11] introduced a system-level realization of CLAHE, which is suitable for very-large-scale integrated (VLSI) implementation. Sasi [12] established an efficient color space for contrast enhancement of heartbeat scent images. In this conclusion, the effects of the histogram equation and the differently defined adaptive histogram equation were explored, and these good development results extended to the appropriate color space. Yussof et al [13] used the CLAHE technique to obtain an enhanced image from a combination of releases performed on the RGB color model and the HSV color model, which was done by Euclidean regulation. Bhairannawar et al [14] proposed the technique of color image enhancement using the laplacian filter and the AHE. Yadav et al [15] used CLAHE enhancement method for improving the video quality in real-time system. Chang et al [16] proposed an automatic CLAHE for image contrast enhancement. Myeongmin et al [33] proposed a novel variational model for the joint enhancement and restoration of low-light images corrupted by blurring and/or noise

Fuzzy set theory has the best ability to deal with uncertain areas in digital image processing. Zadeh [17] proposed this theory in 1965, after which it has been widely used in various fields especially in digital image processing. To improve color images, Hanmandlu et al [18] introduced the Global Variation Extension Operator, which includes three variables, intensification parameter, fuzzifier, and the crossover point. In 2009, Nair et al [19] evaluate the conventional contrast enhancement techniques, and the recent Gray-level ensemble method and fuzzy logic method to find out what is suitable for automatic variation enhancement for ocean satellite images obtained from various sensors. In 2013, Raju et al [20] proposed a fuzzy and histogram-based approach to improving low-intensity color images. The algorithm is faster compared to standard and other new development algorithms. It is based on two important variables. One is $\mathrm{M}$ and the other is $\mathrm{Q}$. Where $\mathrm{M}$ is the mean extreme value of a given image, calculated from the histogram and $\mathrm{k}$ is the contrast amplitude variable. Furthermore, current methods for image en- 
hancement are based on transformational domain methods that may introduce color artifacts and reduce the intensity of input remote sensing images. To overcome this problem, Sharma et al [21] introduced a modified approach, which has the potential to effectively enhance the contrast in digital images using a modified fuzzy-based development algorithm.

Sometimes the membership function of fuzzy sets cannot simultaneously represent evidence of support, opposition, and hesitation. To overcome this problem, Atanassov [22] introduced the higher version of fuzzy sets known as intuitionistic fuzzy sets (IFSs). In 1996, Burillo et al [23] defined the distance between intuitionistic fuzzy sets, and they gave a definitive definition of intuitionistic fuzzy entropy and a theorem that characterizes it. Nowadays several intuitionistic fuzzy c-means clustering algorithm methods were introduced in [24,25] using intuitionistic fuzzy entropy. In this manuscript, we introduce a new approach for color image enhancement algorithm for low light images by using interval-valued intuitionistic fuzzy sets.

This manuscript is arranged as follows. Section 2 outlines the initial stages of the Interval-valued intuitionistic fuzzy set. Section 3 discusses how to create an Interval-valued intuitionistic fuzzy image using Yager's intuitionistic fuzzy generator and also provides different development techniques for low illumination images. Flow chart of the proposed algorithm is given in Fig 1, generation of proposed image is discussed in Fig.2 and Sematic diagram of the bproposed method is given in Fig.??. Section 4, provides results and discussions, which include performance analysis using entropy and correlation coefficient. Finally, the decision is made in Section 5. Images were taken from low light paired dataset (LoL) for testing.

\section{Preliminaries}

\subsection{Fuzzy Sets $(F S s)$}

Let $S=\left\{s_{1}, s_{2}, \ldots, s_{n}\right\}$ be a non-empty set. Define a fuzzy set $B$ of $S$ as

$$
B=\left\{s, \mu_{B}(s) \mid s \in S\right\}
$$

where $\mu_{B}(s): S \rightarrow[0,1]$ represent the degree of belongingness of $s$ in $S$ and we can write the degree of non-belongingness of $s$ in $S$ using the equation $1-\mu_{B}(s)$.

\subsection{Intuitionistic Fuzzy Sets (IFSs)}

An intuitionistic fuzzy set $A$ in $S$, can be express as:

$$
A=\left\{s, \mu_{A}(s), \nu_{A}(s) \mid s \in S\right\}
$$

where $\mu_{A}(s) \rightarrow[0,1], \nu_{A}(s) \rightarrow[0,1]$ are the belongingness and non belongingness degrees of an element $s$ in $A$ with the condition $0 \leq \mu_{A}(s)+\nu_{A}(s) \leq 1$ when $\nu_{A}(s)=1-\mu_{A}(s)$ for every $s$ in $A$, then the set $A$ is said to be an intuitionistic fuzzy set. 
Also consider a degree of hesitation $\pi_{A}(s)$ for all $I F S$ and it is expressed as

$$
\pi_{A}(s)=1-\mu_{A}(s)-\nu_{A}(s)
$$

Here because of the hesitation degree,

$$
\begin{aligned}
& \mu_{A}(s)=1-\pi_{A}(s)-\nu_{A}(s) \\
& \nu_{A}(s)=1-\mu_{A}(s)-\pi_{A}(s)
\end{aligned}
$$

clearly $0 \leq \pi_{A}(s) \leq 1$. So the membership values are in between $\left[\mu_{A}(s), \mu_{A}(s)+\right.$ $\left.\pi_{A}(s)\right]$

2.3 Construction of Intuitionistic Fuzzy Sets

A function $\Psi(s):[0,1] \rightarrow[0,1]$ is called intuitionistic fuzzy generator $[26-28]$ if:

$$
\Psi(s) \leq(1-s) \text { for all } s \in[0,1] \text { and } \Psi(0) \leq 1 \text { and } \Psi(1) \leq 0
$$

In this manuscript, an intuitionistic fuzzy generator is constructed from Yager's generating function [24]. The fuzzy generator function is expressed as

$$
\mathrm{N}_{\mathrm{S}}\left(\mu_{A}(s)\right)=\tau^{-1}\left(\tau(1)-\tau\left(\mu_{A}(s)\right)\right)
$$

where $\tau(\cdot)$ is an increasing function and $\tau:[0,1] \rightarrow[0,1]$.

Yager's class can be generated by using the following function in the above Equation (6):

$$
\tau(s)=s^{\beta}
$$

So, Yager's intuitionistic fuzzy generator can be expressed as: $\mathrm{N}(s)=\left(1-s^{\beta}\right)^{1 / \beta}, \beta>0$ where $\mathrm{N}_{\mathfrak{S}}(1)=0, \mathrm{~N}_{\mathfrak{S}}(0)=1$. Here $\mathrm{N}_{\mathfrak{N}}(s)$ are calculated by Yager's generating function. So IFS will becomes:

$$
A^{I F S}=\left\{s, \mu_{A}(s),\left(1-\mu_{A}(s)^{\beta}\right)^{1 / \beta} \mid s \in S\right\}
$$

and the hesitation degree is

$$
\pi_{A}(s)=1-\mu_{A}(s)-\left(1-\mu_{A}(s)^{\beta}\right)^{1 / \beta}
$$

2.4 Interval-Valued Intuitionistic Fuzzy Sets (IVIFSs)

An IVIFS $\bar{A}$ over $S$ can be express as

$$
\bar{A}=\left\{s, M_{\bar{A}}(s), N_{\bar{A}}(s) \mid s \in S\right\}
$$

where $M_{\bar{A}}(s)$ and $N_{\bar{A}}(s) \subset[0,1]$ are both member and non-member intervals, respectively, and $\sup M_{\bar{A}}(s)+\sup N_{\bar{A}}(s) \leq 1$, for all $s \in S$. 
2.5 Construction of Interval-Valued Intuitionistic Fuzzy Sets

Let us consider the mapping [27]

$$
\varphi: I F S \rightarrow I V I F S
$$

defined as

$$
\varphi(A)=\left\{s, M_{\varphi(A)}(s), N_{\varphi(A)}(s) \mid s \in S\right\}=\bar{A}
$$

where $M_{\varphi(A)}$ and $N_{\varphi(A)}$ are subdivided into the following lower and upper intuitionistic fuzzy interval components.

1. $M_{\varphi(A) L}(s)=M_{\widetilde{A} L}(x)=\mu_{A}(s)-t . \pi_{A}(s), \quad 0 \leq t \leq \frac{\mu_{A}(s)}{\pi_{A}(s)}$.

2. $M_{\varphi(A) U}(s)=M_{\widetilde{A} U}(s)=\mu_{A}(s)+\gamma \cdot \pi_{A}(s), \quad 0 \leq \gamma \leq 1$.

3. $N_{\widetilde{A} L}(s)=\nu_{A}(s)-u \cdot \pi_{A}(s), \quad 0 \leq u \leq \frac{\nu_{A}(s)}{\pi_{A}(s)}$.

4. $N_{\widetilde{A} U}(s)=\nu_{A}(s)+\delta . \pi_{A}(s), \quad 0 \leq \delta \leq 1$,

with $0 \leq \gamma+\delta \leq 1,0<\gamma+t \leq 1$ and $0<\delta+u \leq 1$.

Now define

$$
H_{M}=M_{\widetilde{A} U}(s)-M_{\widetilde{A} L}(s)=(\gamma+t) \cdot \pi_{A}(s)
$$

and

$$
H_{N}=N_{\widetilde{A} U}(s)-N_{\widetilde{A} L}(s)=(\delta+u) \cdot \pi_{A}(s),
$$

then it is obvious that IVIFS is structured so that the width of the member and non-member gap does not exceed its intuitionistic fuzzy index $\left(\pi_{A}\right)$. Here if $A \in F S$, then

$$
\varphi(A)=\left\{s, M_{\varphi(A)}(s), N_{\varphi(A)}(s) \mid s \in S\right\} \text { with } \pi_{A}(s)=0,
$$

which implies

$M_{\widetilde{A} L}(s)=M_{\widetilde{A} U}(s)=\mu_{A}(s)$

$N_{\widetilde{A} L}(s)=N_{\widetilde{A} U}(s)=\nu_{A}(s)$

and hence $\varphi(A)=A$. Therefore, if $A \in F S$ then $\varphi(A)=A$.

\subsection{Histogram Equalization (HE)}

Histogram is graphical representation of any data. Image processing is used to represent the data related to digital images. Histogram is the representation of relative frequency of occurrence of various gray levels. So it represents how many times the gray level value has occurred in a given digital image. Histogram equalization (HE) is a simple and effective image enhancement technique. The gray level of the image is based on the overall density function revisits the grayscale of an image. Because of this, it stretches the dynamic range of an image and enhances image contrast. However, it also tries to change the brightness of an image with an unnatural contrast magnification, for further details see [1]. An algorithm proposed in this paper known as adaptive histogram equalization for an enhancement process and applied over a specific region of any image and adjust contrast according to their neighbor pixels. 
2.7 Adaptive Histogram Equalization (AHE)

The adaptive Histogram equalization [7] is a modified part of the histogram equalization system. In this method, magnification processes are applied to a specific area of an image and adjust the contrast according to their neighboring pixels. This method is only used for uniform fog correction. Here the histogram is divided into some frontal parts. Then adjust the intensity of the area and distribute it evenly over a gray scale image.

\subsection{Contrast Limited Adaptive Histogram Equalization (CLAHE)}

Contrast limited adaptive equalization [15] is the modified part of the adaptive histogram equalization. In this mode, the expansion function is used on all neighboring pixels and the transformation function is obtained. It differs from AHE in that it controls variation. The algorithm of CLAHE is given as follows:

\section{Algorithm 1}

Input: Low contrast color image $I(i, j)$

Output: Contrast limited adaptive histogram-equalized enhanced image $I_{1}(i, j)$ with increased contrast

Step 1: Acquisition process of a low illumination image.

Step 2: Obtain individually all input values used in expansion processes, such as the number of segments in a row and column orientation, the dynamic range (number of pins used in the histogram transformation process), the clip range, and the distribution parameter type.

Step 3: The original image is subdivided and these entries are processed in advance.

Step 4: The process is applied to the tile (contextual region).

Step 5: Create gray level mapping and clipped histogram. At each gray level, the environmental region numbers are evenly distributed in pixels, so the average number of pixels in the gray state is described as follows:

$$
N_{\text {avg }}=\frac{N_{C R-X p} * N_{C R-Y p}}{N_{\text {gray }}}
$$

where

$$
\begin{aligned}
N_{\text {avg }} & =\text { average number of pixels. } \\
N_{\mathrm{CR}-\mathrm{Xp}} & =\text { number of gray level contextual region. } \\
N_{\mathrm{CR}-\mathrm{Yp}} & =\text { number of pixels in } \mathrm{X} \text { direction of contextual region. } \\
N_{\text {gray }} & =\text { number of pixels in } \mathrm{Y} \text { direction of contextual region. }
\end{aligned}
$$

After that calculate the actual clip limit

$$
N_{C L}=N_{C L I P} * N_{a v g}
$$


Step 6: Interrupt gray level mapping to create an enhanced image. Use the fourpixel cluster and apply mapping process in this process, then each mapping tile will be somewhat overlaid on the image area, after which one pixel will be extracted, and then four maps per pixel will be applied. Merge between those results, enhance the pixels, and repeat an image.

\section{Proposed Fuzzy based Method}

\subsection{Interval-Valued Intuitionistic Fuzzy Image (IVIFI)}

Consider an image $I(i, j)$ of $M \times N$ dimension as an array of fuzzy singletons. A crisp image can be modified as a fuzzy image [28] by considering its intensity values as membership values by using the following expression

$$
\mu(I(i, j))=\frac{\xi_{i j}-\xi_{\min }}{\xi_{\max }-\xi_{\min }}
$$

where $\xi_{i j}$ is the pixel value of $(i, j)$ th intensity value. $\xi_{\text {max }}$ and $\xi_{\text {min }}$ denote the maximum and minimum pixel value of the image $I(i, j)$, respectively.

By using equation (13) one can obtain $\nu(I(i, j))$ as follows

$$
\nu(I(i, j))=1-\frac{\xi_{i j}-\xi_{\min }}{\xi_{\max }-\xi_{\min }}
$$

It is usually difficult when selecting a membership values in the fuzzy set. The main goal of the proposed technique is to enhance the given image by eliminating the low illumination values of the member function to the pixels of the uncertain source images. To get rid of them here the fuzzy set is again transformed into the intuitionistic fuzzy set with the third degree (hesitation)

By using Yager's intuitionistic fuzzy generator, $\pi_{A}(s)$ is constructed by using equation (9). From (9) and (13) we can construct an Intuitionistic fuzzy image as

$$
\begin{array}{r}
I F I=\frac{\xi_{i j}-\xi_{\min }}{\xi_{\max }-\xi_{\min }}+\pi_{A}(s) \\
\text { i.e } \quad I F I=\mu(I(i, j))+\pi_{A}(s)
\end{array}
$$

for all $i=1,2, \ldots, m$ and $j=1,2, \ldots, n$

Even though IFS has removed some uncertainties, a number of factors make the image as uncertain again. The value of the selected uncertainty is still an uncertain value. To overcome this problem, IVIFS is used in IFS.

From the expressions of (11) and (12), It is clear that IVIFIs are built so that the width of the member and non-member spaces does not exceed its intuitionistic fuzzy index $\pi_{A}(s)$ : Suppose if If $I_{A}=F S s(I)$, then $\varphi\left(I_{A}\right)=I_{A}$. Finally, IVIFI is produced as below

$$
I V I F I=\mu(I(i, j))+(\gamma+t) \cdot \pi_{A}(s)
$$


where $0<\gamma+t \leq 1$.

$$
\text { i.e } \quad \text { IVIFI }=\mu(I(i, j))+H_{M}
$$

\subsection{Estimation of $(\beta)$ and Hesitation Degrees $\left(H_{M}\right)$}

As shown in Fig.2, the $\beta$ and hesitation degrees $\left(H_{M}\right)$ of equations (9) and (11) are calculated from 0.1 to 1 , by employing entropy values which is described in Section 4.1. Also, amount of $\beta$ and $H_{M}$ are combined together, that gives the most entropy after applying the CLAHE to them i.e., maximum entropy value is taken as the selected image. Here we obtain different $\beta$ and $H_{M}$ values for different images. These combinations of $\beta$ and $H_{M}$ values are automatically generated after completing 100 iterations by using proposed technique.

\subsection{Defuzzification}

Defuzzification is the process of representing a fuzzy set with a crisp number. The same argument is suitable for fuzzified images also. For further information to defuzzification refer [29] and [30]. For defuzzification of intuitionistic fuzzy sets and the crisp image which is get from fuzzified image will become the following expression

$$
\xi_{i j}=\mu(I(i, j)) \cdot\left(\xi_{\max }-\xi_{\min }\right)+\xi_{\min }
$$

hence to defuzzify the interval valued intuitionistic fuzzy image, the expression will becomes

$$
\xi_{i j}=(I V I F I) \cdot\left(\xi_{\max }-\xi_{\min }\right)+\xi_{\min }-H_{M} \cdot\left(\xi_{\max }-\xi_{\min }\right)
$$

where $H_{M}=(\gamma+t) \cdot \pi_{A}(s)$

\subsection{Proposed Algorithm}

Input: Low contrast color image

Output: Contrast enhanced color image

Step 1: Take a color image $I(i, j)$ which is taken in low illumination of size $\mathrm{n}$ $(=M \times N)$.

Step 2: Find fuzzy image $\mu(I(i, j))$ for the given original image $(I(i, j))$ by using the expression (13)

Step 3: Find non-membership degree $\nu_{B}(s)$ for $\mu(I(i, j))$ by using equation (14)

Step 4: By applying Yagers method as described in Section 2.3, one can find $H M 1$ (hesitation) value by taking $\beta$ value as 0.1 .

Step 5: Calculate $H M 11$ by multiplying 0.1 with $H M 1$, the obtained $H M 11$ is adding with Step 2, and then one can get IVIFI1.

Step 6: Construct an enhanced image E1 by applying Algorithm 1 in Step5 and find entropy value for $E 1$. 


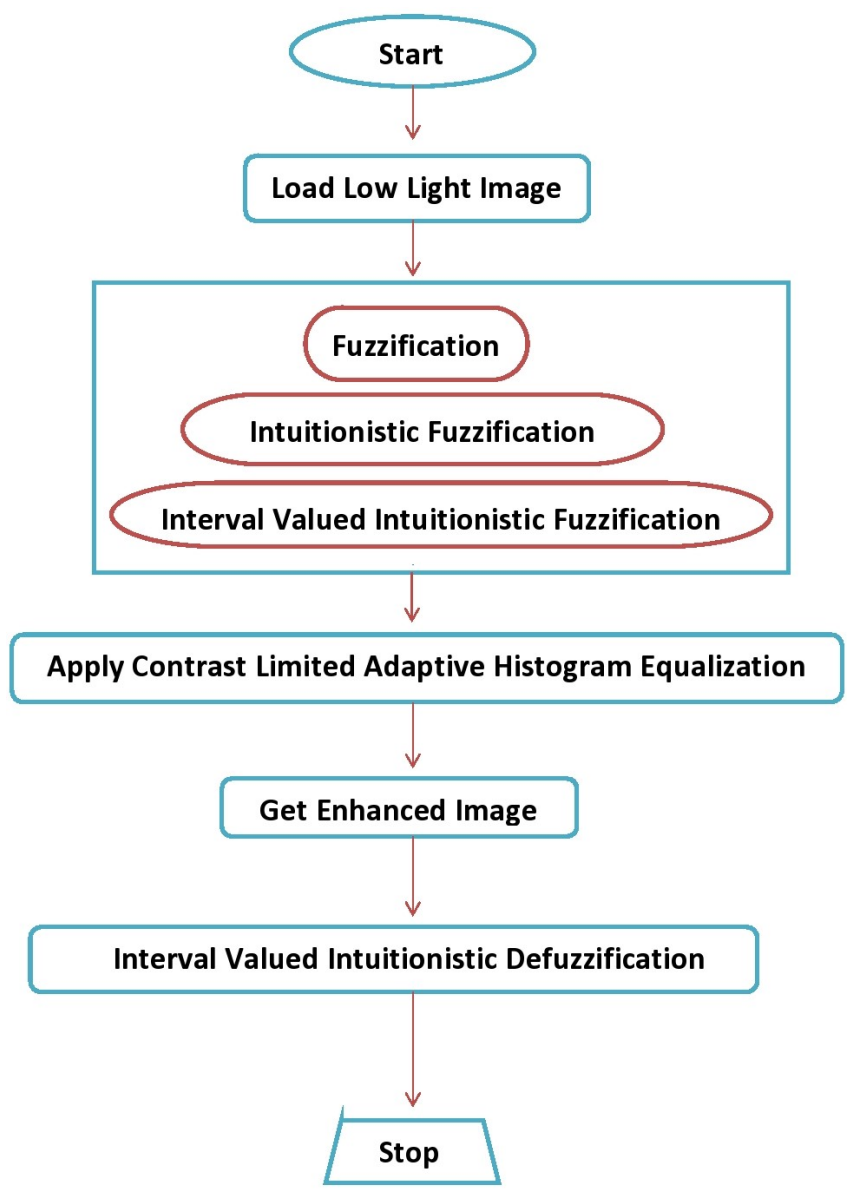

Fig. 1 Flowchart of the Proposed Method

Step 7: Repeat the process from Step 4 to 6 to get $E 2, E 3, . . E 100$.

Step 8: Choose maximum entropy value of image from $E 1$ to $E 100$ which is called our proposed enhanced image after defuzzification as in Section 3.3.

\section{Experiment and Result Analysis}

The experimentation is performed in the environment as follows: The machine CPU is an intel (R) Core (TM) i7-9700 processor that works with a fundamental frequency of $3.00 \mathrm{GHz}$ Quad-Core technology. The hard disk is 2TB, and RAM is 16 GB installed with the Windows 10 Pro operating system's ultimate version. The experimental setup is run over the MATLAB R2018b using the image processing toolbox.

Tests are implemented on 150 images taken from Low Light paired dataset (LoL), seven of which are shown in the first column of Fig.5. The second column 


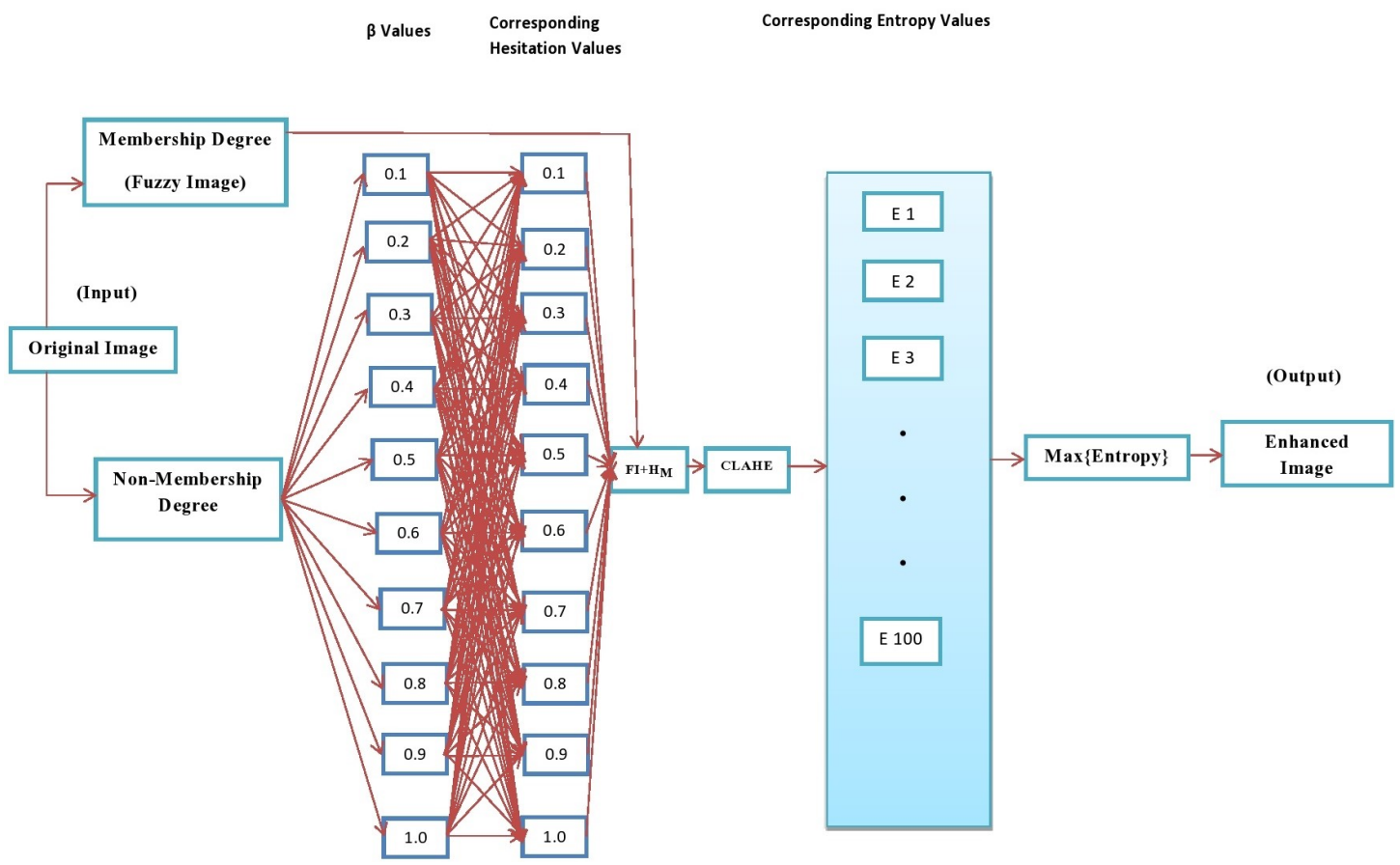

Fig. 2 Generation of Proposed Image

represents the low light images tested by HE method, third column indicates the images enhanced by CLAHE, fourth one is enhanced by BPDFHE [32], Fifth one represent the HSA, sixth column is tested by IFI, seventh is IVIFI and finally eighth one is Proposed method respectively. From Fig.5, we can clearly identify that the proposed method gives better results than other existing literature.

Fig.4 illustrates the original images and their corresponding enhanced images with its $\beta$ and $H_{M}$ values.

The entropy values for the given enhanced methods are discussed in table 1 and their corresponding histogram outputs are discussed in Fig.6. we know that the images with high entropy have the best quality of the image. It shows that the proposed method gives good quality in images. Similarly the correlation coefficient (CC) values for the given enhanced methods are discussed in table 2 and their corresponding histogram output values are discussed in Fig.7. we know that the correlation coefficient [31] is used to measure the size of the correlation between the enhanced and original images. The correlation coefficient has a value of $r=1$ if the two images are completely identical, $r=0$ if they are completely unrelated and $r=-1$ if they are completely anti-correlated. 


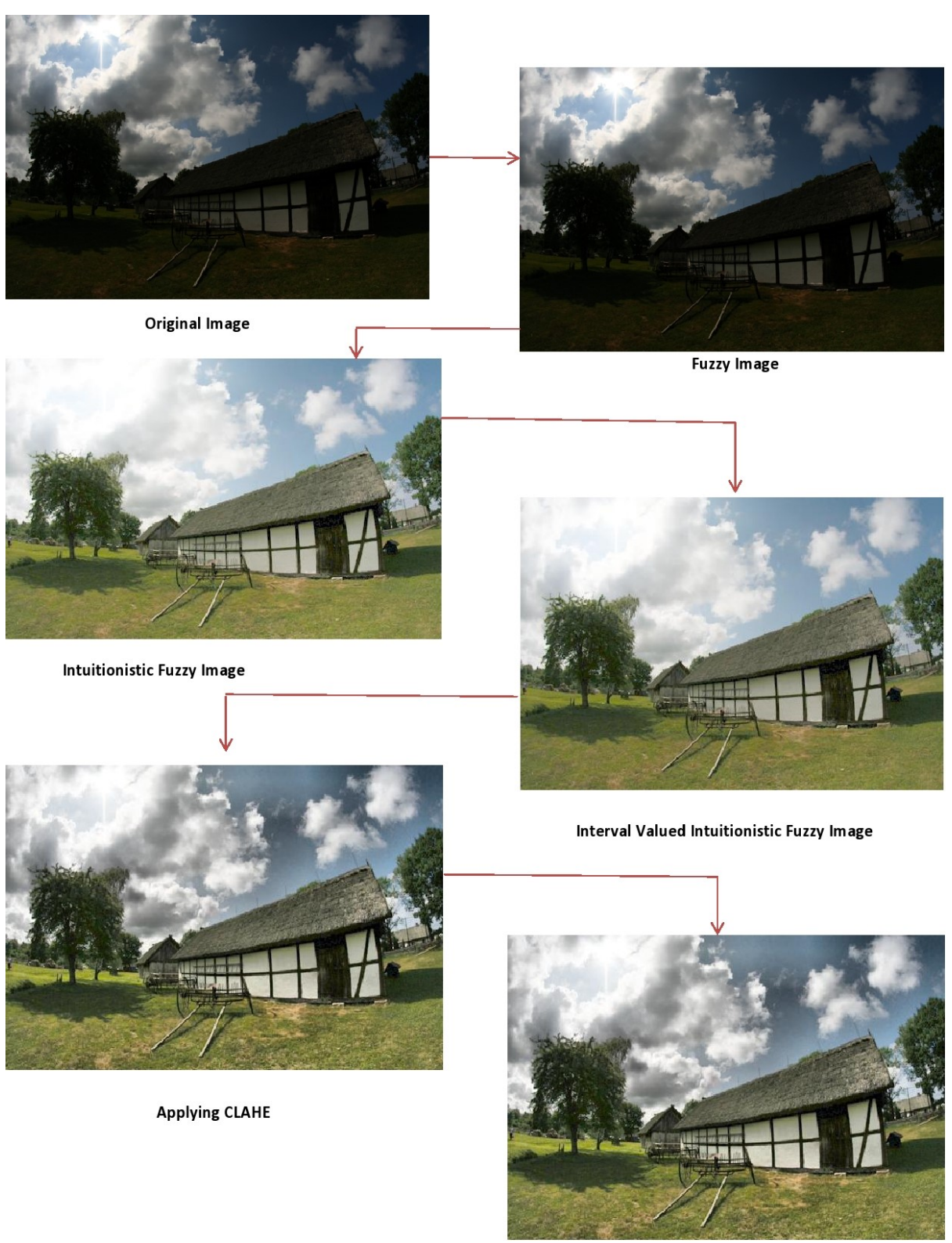

De-Fuzzification (Proposed Method)

Fig. 3 Sematic Diagram of the Proposed Method

\subsection{Performance Analysis}

The quality of an image can be objectively measured using mathematical functions. There are several mathematical functions or measures to evaluate the quality 

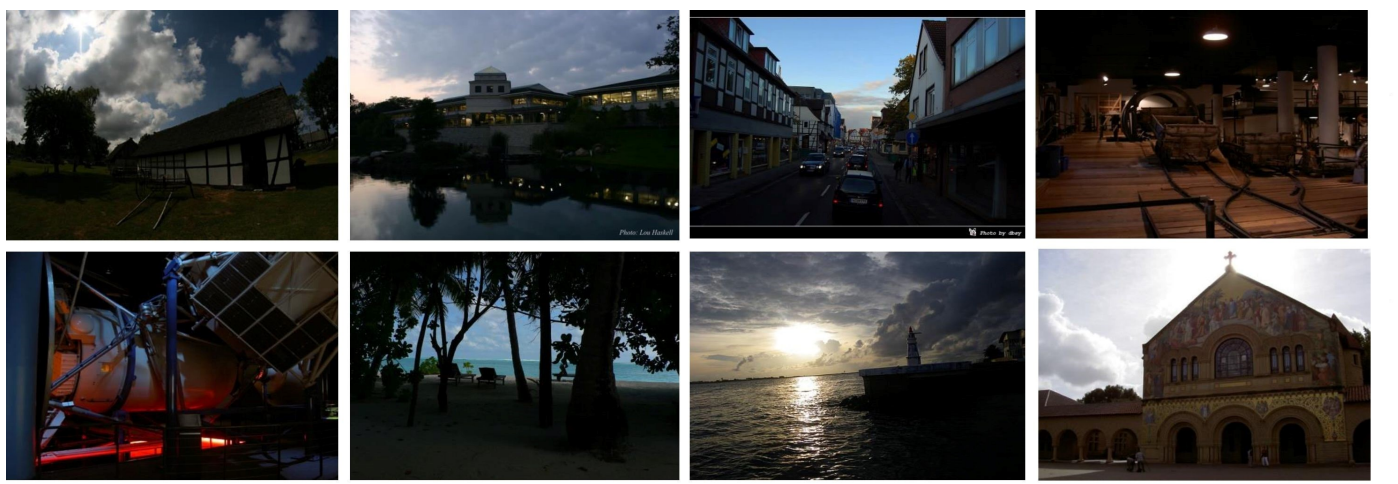

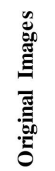
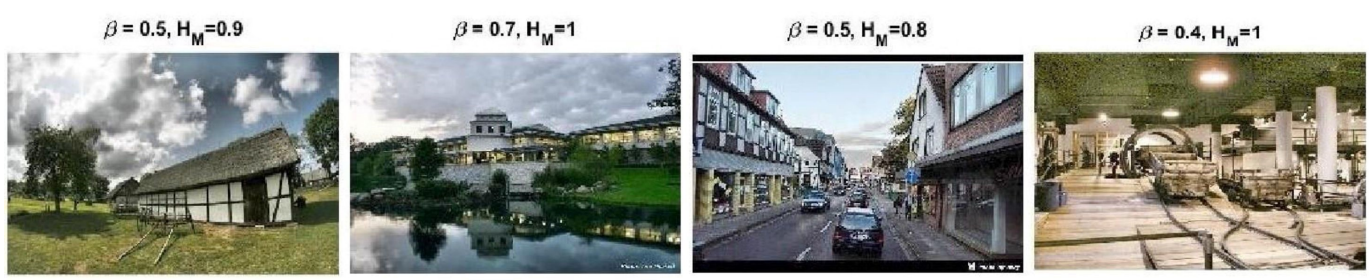

$\beta=0.4, H_{M}=1$

$\beta=0.5, H_{M}=1$

$\beta=0.5, H_{M}=0.9$
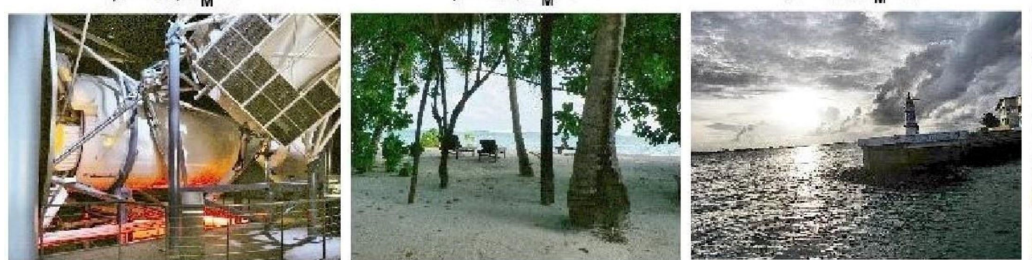

$\beta=0.9, \mathrm{H}_{M}=0.7$

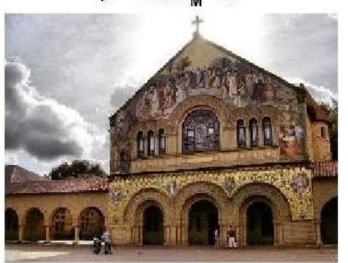

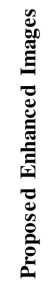

Fig. 4 Proposed Enhanced images with Corresponding $\beta$ and $H_{M}$ Values

of advanced images, such as entropy and correlation coefficients. The method proposed in each performance analysis yields good results.

\subsubsection{Entropy}

Here Shannon entropy [31] with maximum information is used to measure the quality of an image. Expression of entropy value is

$$
E=-\sum_{i=1}^{m} \sum_{j=1}^{n} P(i, j) \log P(i, j)
$$

where $i$ and $j$ represent two different gray-level of the images, $P$ refers the number of co-appearance of gray-levels $i$ and $j$. 


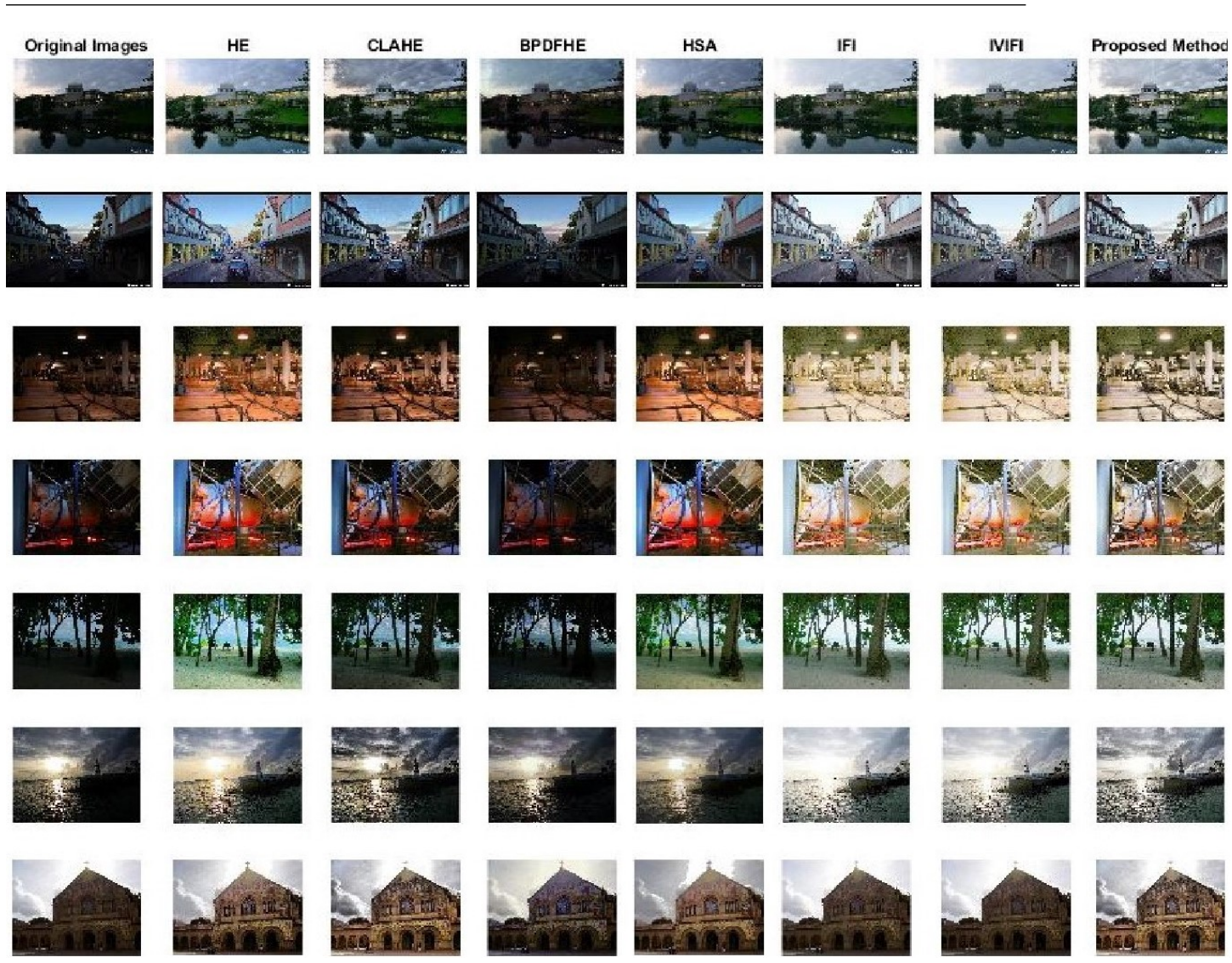

Fig. 5 The Enhanced Output of the Low Light Images comparing with various methods

Table 1 Performance measures using entropy for images in Fig.5

\begin{tabular}{ccccccccc}
\hline S.No & $\begin{array}{c}\text { Original } \\
\text { Image }\end{array}$ & HE & CLAHE & BPDFHE & HSA & IFI & IVIFI $\begin{array}{c}\text { Proposed } \\
\text { Method }\end{array}$ \\
\hline & 7.2662 & 7.4902 & 7.6149 & 7.2405 & 7.5819 & 7.0170 & 7.0170 & $\mathbf{7 . 8 0 2 9}$ \\
2 & 6.7521 & 7.1033 & 7.4047 & 6.7321 & 7.5014 & 6.2245 & 6.4361 & $\mathbf{7 . 6 1 7 7}$ \\
3 & 6.0550 & 6.9248 & 7.1319 & 5.9435 & 7.4615 & 5.8573 & 5.8573 & $\mathbf{7 . 6 4 3 4}$ \\
4 & 6.1372 & 7.0432 & 7.1447 & 5.9295 & 7.4559 & 5.9085 & 5.9085 & $\mathbf{7 . 6 4 7 6}$ \\
5 & 5.8645 & 6.7964 & 6.8424 & 5.6919 & $\mathbf{7 . 5 3 4 6}$ & 5.6317 & 5.6317 & 7.4458 \\
6 & 7.3287 & 7.4547 & 7.6141 & 7.2801 & 7.5312 & 6.7042 & 6.8351 & $\mathbf{7 . 7 8 2 8}$ \\
7 & 7.1763 & 7.3367 & 7.7524 & 7.3240 & 7.4630 & 7.0288 & 7.0836 & $\mathbf{7 . 7 6 3 9}$ \\
\hline
\end{tabular}

4.1.2 Correlation Coefficient $(C C)$

$$
C C=\frac{\sum_{i=1}^{n}\left(x_{i}-\bar{x}\right)\left(y_{i}-\bar{y}\right)}{\sqrt{\sum_{i=1}^{n}\left(x_{i}-\bar{x}\right)^{2}} \sqrt{\sum_{i=1}^{n}\left(y_{i}-\bar{y}\right)^{2}}}
$$




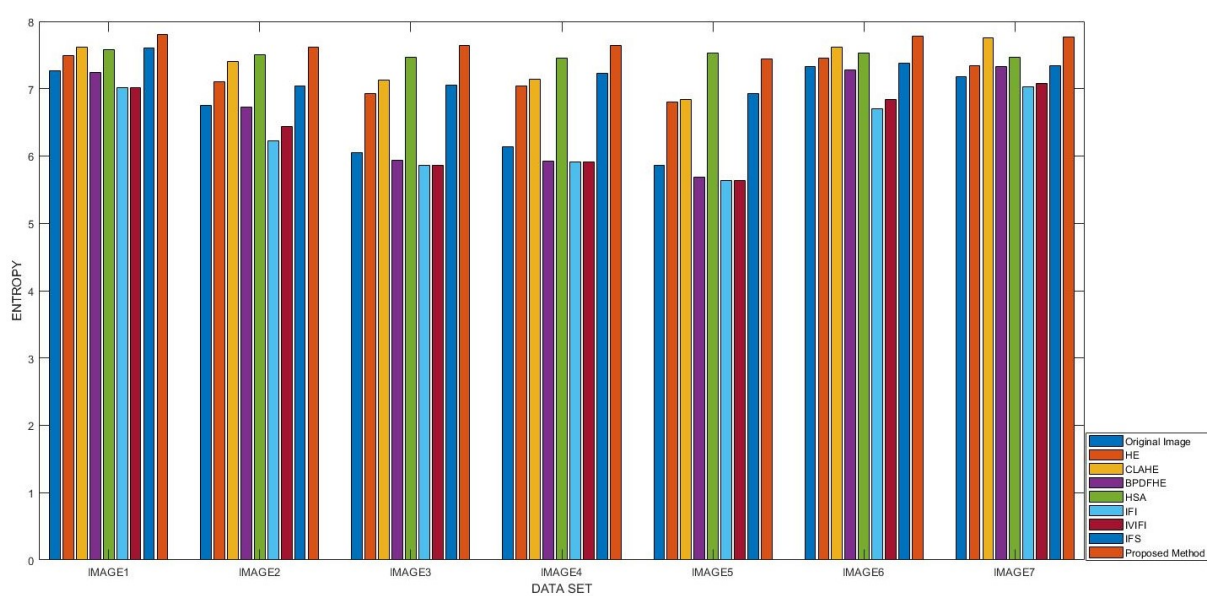

Fig. 6 Histogram output of Entropy values for Fig.5

where $x_{i}, y_{i}$ are the grey values of homologous pixel synthesized image and real high-resolution image and $\bar{x}, \bar{y}$ are mean values of corresponding homologous pixel synthesized image and real high-resolution image.

Table 2 Performance measures using correlation coefficients for images in Fig.5

\begin{tabular}{cccccccc}
\hline $\begin{array}{c}\text { Original } \\
\text { Image }\end{array}$ & HE & CLAHE & BPDFHE & HSA & IFI & IVIFI & $\begin{array}{c}\text { Proposed } \\
\text { Method }\end{array}$ \\
\hline Image 1 & 0.9475 & 0.9177 & 0.9920 & 0.9795 & 0.9770 & 0.9770 & 0.9351 \\
Image 2 & 0.8571 & 0.9096 & 0.9917 & 0.9573 & 0.8648 & 0.9071 & 0.8797 \\
Image 3 & 0.9123 & 0.9275 & 0.9590 & 0.8399 & 0.7939 & 0.7939 & 0.8257 \\
Image 4 & 0.8908 & 0.9334 & 0.9867 & 0.8462 & 0.7689 & 0.7689 & 0.8185 \\
Image 5 & 0.7272 & 0.9579 & 0.9753 & 0.8861 & 0.8790 & 0.8790 & 0.8528 \\
Image 6 & 0.9554 & 0.9003 & 0.9961 & 0.9940 & 0.8593 & 0.8818 & 0.8932 \\
Image 7 & 0.9572 & 0.9047 & 0.9852 & 0.9248 & 0.9986 & 0.9993 & 0.9118 \\
\hline
\end{tabular}

\section{Conclusion and Future Direction}

An efficient interval-valued intuitionistic fuzzy-based color image enhancement method has been proposed. During the test section, the proposed method has been compared with other existing methods such as HE, CLAHE, BPDFHE and HSA. In comparative analysis such as entropy and CC, it is revealed that the proposed method is the best based on quantitative and qualitative improvements. Also, it is more suitable for low light/illumination enhancement for color images than other existing methods. In future the same argument will be utilized in video enhancement techniques. 


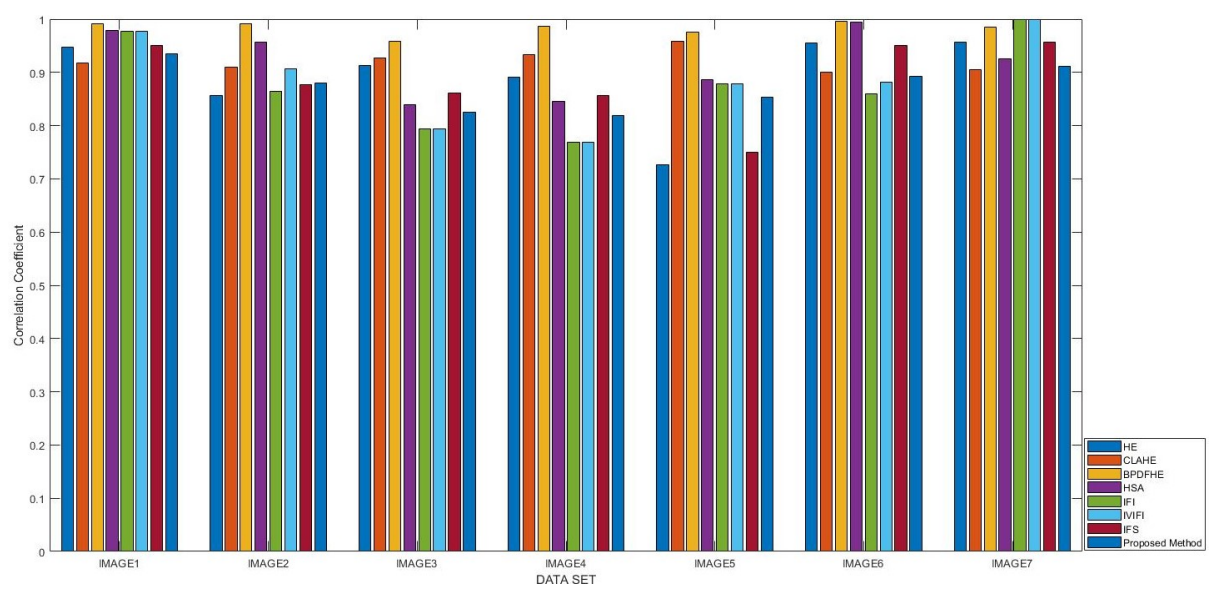

Fig. 7 Histogram output of correlation coefficient values for Fig.5

\subsubsection{Acknowledgment}

This work was supported by University Grants Commission - Special Assistance Program (Department of Special Assistance - I), New Delhi, India, File No. F. 510/7/DSA-1/2015 (SAP-I).

\section{References}

1. FM Abubakar, 'Image enhancement using histogram equalization and spatial filtering', International Journal of Science and Research, 1, 15-20 (2012)

2. Y T Kim, 'Contrast enhancement using brightness preserving bi-histogram equalization', IEEE Transactions on Consumer Electronics, 43, 1-8, (1997)

3. S D Chen and A R Ramli, 'Minimum mean brightness error bi-histogram equalization in contrast enhancement', IEEE Transactions on Consumer Electronics, 49, 1310-1319, (2003)

4. H Ibrahim and N S P Kong, 'Brightness preserving dynamic histogram equalization for image contrast enhancement', IEEE Transactions on Consumer Electronics, 53, 1752-1758 (2007)

5. K Kapoor and S Arora, 'Color image enhancement based on histogram equalization', Electrical and Computer Engineering, 4, 73-82, (2015)

6. S D Chen, A R Ramli,'Preserving brightness in histogram equalization based contrast enhancement techniques', Digital Signal Processing, 14, 413-428 (2004)

7. S M Puer, E P Amburn, J D Austin, R Cromartie, A Geselowit, T Greer, B T H Romeny, J B Zimmerman and K Zuiderveld, 'Adaptive histogram equalization and its variations', Computer Vision Graphics and Image processing, 39, 1-22 (1986)

8. G Jianga, S C F Lina, C Y Wonga, M A Rahmana, T R Rena, N Kwoka, H Shi, Y H $\mathrm{Yu}$ and $\mathrm{T} \mathrm{Wu}$, 'Color image enhancement with brightness preservation using a histogram specification approach', Optik, 126, 5656-5664 (2015)

9. J Patia and M Ogate, 'A novel image enhancement technique based on statistical analysis of DCT coefficients for JPEG compressed images', IEEE Explore, Twenty-Second National Conference on Communication (NCC), 978, 2361-5 (2016)

10. E D Pisano, S Zong, B M Hemminger, M Deluca, R E Johnston, K Muller, M P Braeuning and S M Pizer, 'Contrast limited adaptive histogram equalization image processing to improve the detection of simulated spiculations in dense mammograms', Journal of Digital Imaging, 11, 193-200 (1998)

11. A M Reza, 'Realization of the contrast limited adaptive histogram equalization (CLAHE) for real-time image enhancement', Journal of VLSI Signal Processing, 38, 3544, (2004) 
12. N M Sasi, V K Jayasree,'Contrast limited adaptive histogram equalization for qualitative enhancement of myocardial perfusion images', Scientific Research, 5, 326-331 (2003)

13. W N Yussof, M S Hitam, E A Awalludin, and Z Bachok, 'Performing contrast limited adaptive histogram equalization technique on combined color models for underwater image enhancement', International Journal of Interactive Digital Media, 1, 2289-4101 (2013)

14. S Bhairannawar, A Patil, A Janmane, M Huilgol, 'Color image enhancement using laplacian filter and contrast limited adaptive histogram equalization', IEEE Explore, International Conference on Innovations in Power and Advanced Computing Technologies, 978, 5682-8, (2017)

15. G Yadav S Maheshwari A Agarwal 'Contrast limited adaptive histogram equalization based enhancement for real-time video system, IEEE Explore, International Conference on Advances in Computing, Communications and Informatics, 978, 3080-7 (2014)

16. Y Chang $\mathrm{C}$ Jung, $\mathrm{P} \mathrm{Ke}, \mathrm{H}$ Song and J Hwang 'Automatic contrast limited adaptive histogram equalization with dual gamma correction', IEEE Access, 6, 11782 - 11792 (2018) 17. A Zadeh, 'Fuzzy Sets', Information and Control, 8, 338-353 (1965)

18. M Hanmandlu and D Jha, 'An optimal fuzzy system for color image enhancement', IEEE Transactions on Image Processing, 15, 2956-2966 (2006)

19. M S Nair R Lakshmanan M Wilscy and R Tatavarti, 'Fuzzy logic-based automatic contrast enhancement of satellite images of ocean', Springer-Verlag London Limited, 5, 69-80 (2009)

20. G Raju and M S Nair, 'A fast and efficient color image enhancement method based on fuzzy-logic and histogram', International Journal of Electronics and Communications 68, 237-243 (2014)

21. S Sharma and A Bhatia, 'Contrast enhancement of an image using fuzzy logic', International Journal of Computer Applications, 111, 0975-8887 (2015)

22. K T Atanassov, 'Intuitionistic fuzzy sets', Fuzzy Sets and Systems, 20, 8796 (1986)

23. $\mathrm{P}$ Burillo and $\mathrm{H}$ Bustince, 'Entropy on intuitionistic fuzzy sets and on interval-valued fuzzy sets', Fuzzy Sets and Systems, 78, 305-316 (1996)

24. T Chaira, 'A novel intuitionistic Fuzzy c-means clustering algorithm and its application to medical images', Applied Soft Computing, 11, 1711-1717 (2011)

25. P Balasubramaniam, V P Ananthi, 'Segmentation of nutrient deficiency in incomplete crop images using intuitionistic fuzzy c-means clustering algorithm', Nonlinear Dynamics, 83, 849-866 (2015)

26. H Bustince, J Kacprzyk and V Mohedano, 'Intuitionistic fuzzy generators: application to intuitionistic fuzzy complementation', Fuzzy Sets and Systems 114, 485-504 (2000)

27. V P Ananthi, $\mathrm{P}$ Balasubramaniam and T Kalaiselvi, 'A new fuzzy clustering algorithm for the segmentation of brain tumor', Soft Computing: Methodologies and Applications, 20, 48594879 (2016)

28. P Balasubramaniam and V P Ananthi, 'Image fusion using intuitionistic fuzzy sets', Information Fusion, 20, 21-30 (2014)

29. P Mittal, R K Saini and N K Jain, 'Image enhancement using fuzzy logic techniques', Softcomputing:Theories and Applications, 742, 537-546 (2018)

30. C Radhika and R Parvathi, 'Defuzzification of intuitionistic fuzzy sets', Notes on Intuitionistic Fuzzy Sets, 22, 1926 (2016)

31. S S Somvanshi, P Kunwar, S Tomar and M Singh, 'Comparative statistical analysis of the quality of image enhancement techniques', International Journal of Image and Data Fusion, 9, 131-151 (2017)

32. D Sheet and A Suveer, 'Brightness Preserving Dynamic Fuzzy Histogram Equalization', IEEE Transactions on Consumer Electronics, 56, 2475-2480 (2010)

33. M Kang and M Jung, 'Simultaneous image enhancement and restoration with non-convex total variation', Journal of Scientific Computing, 83, 1-46 (2021) 